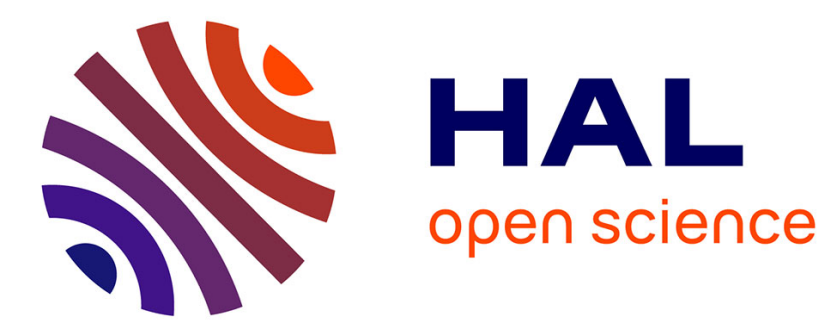

\title{
Gain-Scheduled Autopilot Design with Anti-Windup Compensator for a Dual-Spin Canard-Guided Projectile
}

Sovanna Thai, Spilios Theodoulis, Clement Roos, Jean-Marc Biannic, Michael Proff

\section{- To cite this version:}

Sovanna Thai, Spilios Theodoulis, Clement Roos, Jean-Marc Biannic, Michael Proff. Gain-Scheduled Autopilot Design with Anti-Windup Compensator for a Dual-Spin Canard-Guided Projectile. 2020 IEEE Conference on Control Technology and Applications (CCTA), Aug 2020, Montreal, France. pp.156-161, 10.1109/CCTA41146.2020.9206311 . hal-03003964

\section{HAL Id: hal-03003964 https://hal.science/hal-03003964}

Submitted on 13 Nov 2020

HAL is a multi-disciplinary open access archive for the deposit and dissemination of scientific research documents, whether they are published or not. The documents may come from teaching and research institutions in France or abroad, or from public or private research centers.
L'archive ouverte pluridisciplinaire HAL, est destinée au dépôt et à la diffusion de documents scientifiques de niveau recherche, publiés ou non, émanant des établissements d'enseignement et de recherche français ou étrangers, des laboratoires publics ou privés. 


\title{
Gain-Scheduled Autopilot Design with Anti-Windup Compensator for a Dual-Spin Canard-Guided Projectile
}

\author{
Sovanna Thai ${ }^{1}$, Spilios Theodoulis ${ }^{1}$, Clément Roos ${ }^{2}$, Jean-Marc Biannic ${ }^{2}$, and Michael Proff ${ }^{1}$
}

\begin{abstract}
This paper explores the design of an autopilot for a dual-spin guided projectile taking into account input saturations. The projectile full nonlinear model is described and then put in a quasi-LPV form suitable for controller synthesis. A baseline gain-scheduled autopilot is then computed without taking into account the saturation nonlinearities. As a major contribution of this paper, the impact of saturations is next highlighted through a degraded flight scenario, and an antiwindup compensator is added to the closed-loop to counteract their effects. The improvements brought by the anti-windup are then evaluated through Monte Carlo simulations.
\end{abstract}

Index Terms-gain-scheduling, autopilot design, antiwindup, guided projectiles

\section{INTRODUCTION}

Standard ballistic projectiles suffer from a lack of accuracy that severely restricts the achievable operational requirements. Guided projectiles constitute a solution to overcome these limitations and fulfil more stringent specifications. Many guided projectile concepts integrate steering mechanisms in the form of additional aerodynamic surfaces to be manipulated during the flight. The concept studied in the present paper is that of a dual-spin projectile whose nose, decoupled from the body, is equipped with four independently actuated canards that can be deflected to alter the trajectory (see Fig. 1). Previous works on this concept have been conducted on both the modelling and control of such a system ([1], [2], [3]) without explicitly taking saturations into account. The aim of the current paper is twofold. The first objective is to propose an alternative approach to the baseline autopilot design for the picth/yaw axes, with the aim to streamline this phase of the study. The second objective, which constitutes the main contribution of the paper, is to address the issue of saturations.

This paper is organised as follows. Section II recalls the projectile nonlinear and linearised models. Section III then describes the adopted methodology for the baseline autopilot design, and introduces the saturation problem by providing a motivating scenario in which the incurred performance loss is clearly unacceptable in an operational context. This problem is addressed more directly in Section IV, where the main principles of anti-windup are reminded and an anti-windup compensator is computed. Finally, Section V details Monte

\footnotetext{
${ }^{1}$ Sovanna Thai, Spilios Theodoulis and Michael Proff are with the Guidance, Navigation \& Control Department of the French-German Research Institute of Saint-Louis (ISL), Saint-Louis,France <sovanna.thai, spilios.theodoulis, michael.proff>eisl.eu

${ }^{2}$ Clément Roos and Jean-Marc Biannic are with the Information Processing and Systems Department of ONERA, The French Aerospace Lab, Toulouse, France <clement.roos, jean-marc.biannic>@onera.fr
}

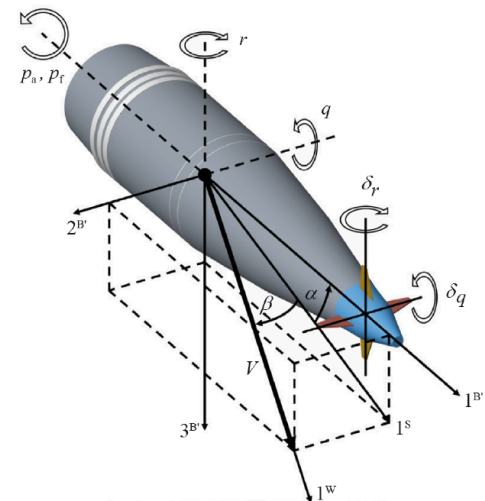

Fig. 1. Dual-spin projectile concept (forward part in blue, aft part in grey)

Carlo simulations that showcase the improvements brought by the anti-windup compensator.

\section{Airframe Modelling}

\section{A. Nonlinear 7-DoF Model}

The nonlinear model of the projectile is composed of translational and attitude dynamic equations resulting from Newton's and Euler's laws. These equations are given in a non-rolling reference frame $B^{\prime}$ as in [4]:

$$
\begin{aligned}
& {\left[\begin{array}{c}
\dot{u} \\
\dot{v} \\
\dot{w}
\end{array}\right]=\frac{1}{m}\left[\begin{array}{c}
X \\
Y \\
Z
\end{array}\right]-\left[\begin{array}{ccc}
0 & -r & q \\
r & 0 & r \tan \theta \\
-q & -r \tan \theta & 0
\end{array}\right]\left[\begin{array}{c}
u \\
v \\
w
\end{array}\right]} \\
& {\left[\begin{array}{c}
\dot{p}_{f} \\
\dot{p}_{a} \\
\dot{q} \\
\dot{r}
\end{array}\right]=\left[\begin{array}{c}
I_{x f}{ }^{-1} L_{f} \\
I_{x a}{ }^{-1} L_{a} \\
I_{t}{ }^{-1} M \\
I_{t}{ }^{-1} N
\end{array}\right]+\left[\begin{array}{c}
0 \\
-\left(I_{x a} I_{t}{ }^{-1} p_{a}+r \tan \theta\right) r \\
\left(I_{x a} I_{t}{ }^{-1} p_{a}+r \tan \theta\right) q
\end{array}\right]}
\end{aligned}
$$

where the dynamic states are the linear velocities $(u, v, w)$ of the projectile and the angular rates $\left(p_{f}, p_{a}, q, r\right)$ of both the forward $(f)$ and aft $(a)$ projectile parts. The constants appearing in this equation are the mass $m$ of the projectile, and the moments of inertia $I_{x f}, I_{x a}, I_{t}$. This description of the system is complemented with translational and attitude kinematic equations describing the linear $\left(x_{e}, y_{e}, z_{e}\right)$ and angular $\left(\phi_{f}, \phi_{a}, \theta, \psi\right)$ positions of the projectile with respect to the inertial reference frame, given by [5]:

$$
\begin{aligned}
& {\left[\begin{array}{c}
\dot{x}_{e} \\
\dot{y}_{e} \\
\dot{z}_{e}
\end{array}\right]=\left[\begin{array}{ccc}
\cos \psi \cos \theta & -\sin \psi & \cos \psi \sin \theta \\
\sin \psi \cos \theta & \cos \psi & \sin \psi \sin \theta \\
-\sin \theta & 0 & \cos \theta
\end{array}\right]\left[\begin{array}{c}
u \\
v \\
w
\end{array}\right]} \\
& {\left[\begin{array}{c}
\dot{\phi}_{f} \\
\dot{\phi}_{a} \\
\dot{\theta} \\
\dot{\psi}
\end{array}\right]=\left[\begin{array}{cccc}
1 & 0 & 0 & \tan \theta \\
0 & 1 & 0 & \tan \theta \\
0 & 0 & 1 & 0 \\
0 & 0 & 0 & \sec \theta
\end{array}\right]\left[\begin{array}{c}
p_{f} \\
p_{a} \\
q \\
r
\end{array}\right]}
\end{aligned}
$$


The external forces $X, Y, Z$ include drag/lift, Magnus (originating from the spinning motion), control (generated by the canards), and gravitational terms. The external moments $L_{f}, L_{a}, M, N$ consist of pitch/yaw, Magnus, damping, control, and front-aft friction terms. Their expressions involve aerodynamic coefficients that depend in a nonlinear manner on the Mach number $\mathcal{M}=V / a(h)$, with $V$ the airspeed and $a$ the altitude-dependent speed of sound, and for some also on the aerodynamic angles of attack (AoA $\alpha$ ) and sideslip $(\operatorname{AoS} \beta)$. Due to space constraints, we refer to [3] for their detailed expressions. The control terms depend on variables $\delta_{p}, \delta_{q}$, and $\delta_{r}$, which are virtual control variables that are function of the nose roll angle $\phi_{f}$ and on the deflection angles of the four canards $\delta_{1}, \ldots, \delta_{4}$ through the relation:

$$
\left[\begin{array}{l}
\delta_{p} \\
\delta_{q} \\
\delta_{r}
\end{array}\right]=T\left(\phi_{f}\right)\left[\begin{array}{cccc}
\frac{1}{4} & \frac{1}{4} & \frac{1}{4} & \frac{1}{4} \\
0 & \frac{1}{2} & 0 & -\frac{1}{2} \\
\frac{1}{2} & 0 & -\frac{1}{2} & 0
\end{array}\right]\left[\begin{array}{c}
\delta_{1} \\
\delta_{2} \\
\delta_{3} \\
\delta_{4}
\end{array}\right]
$$

with $T\left(\phi_{f}\right)=\left[\begin{array}{ccc}1 & 0 & 0 \\ 0 & \cos \phi_{f} & -\sin \phi_{f} \\ 0 & \sin \phi_{f} & \cos \phi_{f}\end{array}\right]$

The aerodynamic variables $V, \alpha$ and $\beta$ are related to the linear velocities (under a no-wind assumption) through:

$$
\begin{aligned}
& V=\sqrt{u^{2}+v^{2}+w^{2}} \\
& \alpha=\arctan \left(\frac{w}{u}\right) \quad \beta=\arctan \left(\frac{v}{\sqrt{u^{2}+w^{2}}}\right)
\end{aligned}
$$

These relations allow (see e.g. [3]) to rewrite the translational dynamic equation (1a) in terms of the states $(V, \alpha, \beta)$, which is more suitable for autopilot design:

$$
\begin{aligned}
& {\left[\begin{array}{c}
\dot{V} \\
\dot{\alpha} \\
\dot{\beta}
\end{array}\right]=\left[\begin{array}{c}
0 \\
q+r(\cos \alpha \tan \theta-\sin \alpha) \tan \beta \\
-r(\cos \alpha+\sin \alpha \tan \theta)
\end{array}\right]} \\
& +\frac{1}{m V}\left[\begin{array}{ccc}
V \cos \alpha \cos \beta & V \sin \beta & V \sin \alpha \cos \beta \\
-\sin \alpha / \cos \beta & 0 & \cos \alpha / \cos \beta \\
-\cos \alpha \sin \beta & \cos \beta & -\sin \alpha \sin \beta
\end{array}\right]\left[\begin{array}{l}
X \\
Y \\
Z
\end{array}\right]
\end{aligned}
$$

\section{B. Pitch/Yaw Dynamics Linearised Model}

In the context of conceiving the autopilot of the projectile, the states of interest are those pertaining to the projectile nose $\left(p_{f}, \phi_{f}\right)$ on one hand, and to the highly coupled pitch and yaw axes $(\alpha, q, \beta, r)$ on the other hand. The aim of the roll-channel autopilot is to stabilise the roll angle $\phi_{f}$ around a fixed value, typically either $0^{\circ}$ or $45^{\circ}$, as a prerequisite before considering the pitch and yaw axes. The modelling and autopilot design for the roll-channel are addressed in [6]. Regarding the pitch/yaw channels, the relevant nonlinear dynamics can be rewritten in the generic parameter-dependent form

$$
\begin{aligned}
\dot{\boldsymbol{x}}(t) & =f(\boldsymbol{x}(t), \boldsymbol{u}(t), \boldsymbol{\sigma}(t)) \\
\boldsymbol{y}(t) & =g(\boldsymbol{x}(t), \boldsymbol{u}(t), \boldsymbol{\sigma}(t))
\end{aligned}
$$

with states $\boldsymbol{x}=[\alpha, q, \beta, r]^{T}$, inputs $\boldsymbol{u}=\left[\delta_{q}, \delta_{r}\right]^{T}$, outputs $\boldsymbol{y}=\left[n_{z}, n_{y}, q, r\right]^{T}$, with $\left[n_{z}, n_{y}\right]=\frac{1}{m g}\left[Z_{s}, Y_{s}\right]$ the normal and lateral load factors (defined as the contribution of the external forces excluding gravity), and parameters $\sigma=$
$\left[V, h, p_{a}, \theta\right]^{T}$. The set of values taken by the parameter vector $\sigma$ defines the flight envelope, which can be approximated as a hyperrectangle $\boldsymbol{\Gamma}_{\boldsymbol{\sigma}} \subset \mathbb{R}^{4}$ whose bounds can be estimated through ballistic trajectories covering all possible launch conditions.

An extended trimming vector $\boldsymbol{\rho}=\left[V, h, p_{a}, \theta, \alpha, \beta\right]^{T}$, taking its values in an extended flight envelope $\Gamma_{\rho} \subset$ $\mathbb{R}^{6}$, is defined to compute equilibrium points. A Jacobian linearisation can then be performed for each value of $\rho$ in the flight envelope, resulting in a quasi-LPV model of the form

$$
\begin{aligned}
& \dot{\boldsymbol{x}}_{\epsilon}(t)=\boldsymbol{A}(\boldsymbol{\rho}) \boldsymbol{x}_{\epsilon}(t)+\boldsymbol{B}(\boldsymbol{\rho}) \boldsymbol{u}_{\epsilon}(t) \\
& \boldsymbol{y}_{\epsilon}(t)=\boldsymbol{C}(\boldsymbol{\rho}) \boldsymbol{x}_{\epsilon}(t)+\boldsymbol{D}(\boldsymbol{\rho}) \boldsymbol{u}_{\epsilon}(t)
\end{aligned}
$$

with $\boldsymbol{x}_{\epsilon}, \boldsymbol{u}_{\epsilon}$ and $\boldsymbol{y}_{\epsilon}$ the state, input and output deviation vectors from equilibrium. The trimming algorithm and expressions of the state-space matrices are detailed in [1] and are not recalled here for brevity.

The open-loop model used for autopilot design consists of the quasi-LPV model augmented with actuators and sensors. The dynamics of the latter are neglected for this study. The canard actuators are modelled as second-order systems with natural frequency $\omega_{\delta}=2 \pi \cdot 20 \mathrm{rad} / \mathrm{s}$ and damping ratio $\xi_{\delta}=$ 0.781 , with an additional amplitude saturation $\delta_{\text {sat }}= \pm 10^{\circ}$ on the $\delta_{i}, i=1 \ldots 4$.

\section{BAseline Autopilot Design}

Controller synthesis for a system with input constraints is classically done by initially ignoring these constraints, in a first design phase that results in a so-called baseline autopilot. It is then checked through closed-loop simulations whether the saturation levels are reached on the scenarios of interest. If saturations occur and result in a breach of the operational requirements, the design of the controller is revised to reduce the impact of the saturations. This section presents the first phase of this two-step procedure while proposing an alternative approach to the design method used in [1] or [3].

\section{A. General Methodology}

Obtaining a gain-scheduled controller is typically done by interpolating local controllers computed for different operating points [7]. Despite the lack of theoretical guarantees that can be obtained regarding global stability and performance, this method remains highly relevant due to its ease of implementation and understanding, good behaviour observed in practice, and low level of conservatism.

To alleviate the computational burden and reduce the number of syntheses, it is interesting to consider a trim vector of reduced dimension. A sensible choice of the parameters to retain can be made by performing a simulation-based sensitivity analysis as described in [2]. The reduced trim vector chosen in this study is defined as $\boldsymbol{\lambda}=\left[V, h, p_{a}\right]^{T}$, thus defining a three-dimensional flight envelope described by the intervals $V \in[140 \mathrm{~m} / \mathrm{s}, 420 \mathrm{~m} / \mathrm{s}], h \in[0 \mathrm{~m}, 15000 \mathrm{~m}]$, and $p_{a} \in[750 \mathrm{rad} / \mathrm{s}, 1600 \mathrm{rad} / \mathrm{s}]$, with the remaining parameters fixed to their median values. An $8 \times 6 \times 5$ grid is considered, 
amounting to 240 synthesis points. The global controller is then obtained by linear interpolation.

\section{B. Controller Structure and Synthesis}

The proposed fixed-structure controller is presented in Fig. 2, the subscripts $c$ and $m$ denoting commanded signals and measurements respectively. To lighten notations, the subscript $\epsilon$ signifying distance to equilibrium is omitted. Symmetries of the airframe model are exploited by imposing corresponding symmetries on the gain matrices of the controller, further reducing the interpolation and implementation effort.

$$
\begin{aligned}
\mathbf{K}_{i, e}=\left[\begin{array}{cc}
K_{i, e}^{(11)} & K_{i, e}^{(12)} \\
K_{i, e}^{(12)} & -K_{i, e}^{(11)}
\end{array}\right] & \mathbf{K}_{p, n}=\left[\begin{array}{cc}
K_{p, n}^{(11)} & K_{p, n}^{(12)} \\
K_{p, n}^{(12)} & -K_{p, n}^{(11)}
\end{array}\right] \\
\mathbf{K}_{p, \omega}=\left[\begin{array}{cc}
K_{p, \omega}^{(11)} & K_{p, \omega}^{(12)} \\
-K_{p, \omega}^{(12)} & K_{p, \omega}^{(11)}
\end{array}\right] & \mathbf{K}_{p, \delta}=\left[\begin{array}{cc}
K_{p, \delta}^{(11)} & K_{p, \delta}^{(12)} \\
K_{p, \delta}^{(12)} & -K_{p, \delta}^{(11)}
\end{array}\right]
\end{aligned}
$$

The eight controller gains are computed by solving an $\mathcal{H}_{\infty}$ controller synthesis problem using the MATLAB command systune [8]. The standard plant-controller interconnection is augmented with weighting filters to express closed-loop requirements in the frequency domain, based on a mixedsensitivity approach [9]. A key aspect to address is the parametrisation of the tuning goals at each synthesis point. Instead of adjusting the weighting filters as is done in [3], the adopted approach consists in scaling the linear airframe model $G(s)$ by a matrix gain $U$ so that the DC gain of $G_{\text {scaled }}(s)=U \cdot G(s)$ is unitary. The gains provided by systune correspond then to a controller $K_{\text {scaled }}(s)=$ $K(s) \cdot U^{-1}$ tuned for the scaled closed-loop, from which the gains to implement $K(s)$ can be obtained.

A synthesis point corresponding to high velocity and low altitude is then chosen as the basis to formulate the controller synthesis problem. Hard tuning goals for lowfrequency disturbance rejection and high-frequency control signal attenuation are specified as:

$$
\begin{array}{r}
\left\|W_{S}(s) T_{n_{z, c} \rightarrow e_{z}}(s)\right\|_{\infty}<1 \\
\left\|W_{S}(s) T_{n_{y, c} \rightarrow e_{y}}(s)\right\|_{\infty}<1 \\
\left\|W_{K S}(s) T_{\left[\begin{array}{l}
n_{z, c} \\
n_{y, c}
\end{array}\right] \rightarrow\left[\begin{array}{c}
\delta_{q} \\
\delta_{r}
\end{array}\right]}(s)\right\|_{\infty}<1
\end{array}
$$

with $W_{S}(s)=\frac{s / 2+4}{s+4 \cdot 10^{-4}}$ and $W_{K S}(s)=W_{K S}=1 / 3$. In addition, stability margins of $6 \mathrm{~dB}$ and $30^{\circ}$ are imposed at controller input and output. The selected soft goal is a step tracking goal expressed in the time-domain [10], with a second-order system of natural frequency $\omega=5 \mathrm{rad} / \mathrm{s}$ and damping ratio $\zeta=0.78$ as a reference model, corresponding to an overshoot of $2 \%$ and a settling time of $0.72 \mathrm{~s}$.

Fig. 3 and 4 show the tuning results obtained for the chosen design point. Referring to Fig. 3, it is notable that good controller roll-off is achieved despite $W_{K S}$ being chosen as a constant. The step responses show good behaviour in the time-domain as well, with an efficient decoupling of the normal and lateral axes. The achieved performance index is $\gamma=2.67$. The above problem is then solved for all 240 points of the design grid. With no adjustments of the

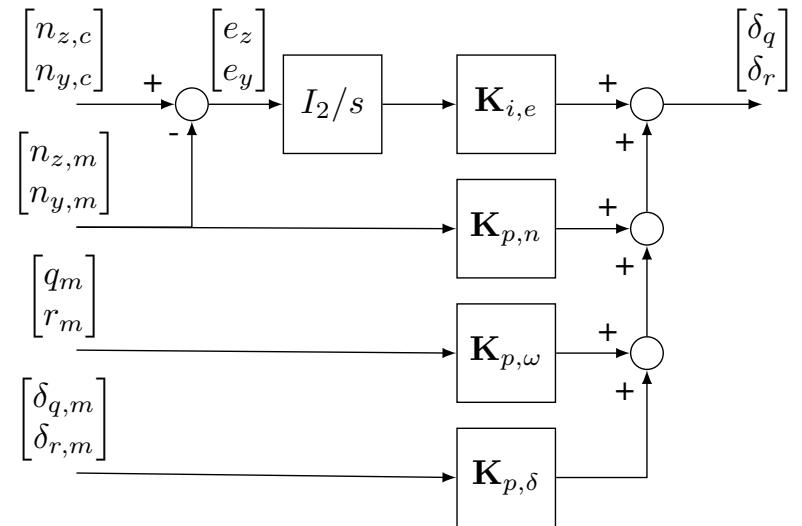

Fig. 2. I-P structure of the pitch/yaw channel linear controller

tuning goals, a worst case performance index of $\gamma=3.54$ is obtained for the soft goal, while the hard goals remain satisfied all across the design grid, showcasing the efficiency of plant scaling.

\section{Validation on a Nominal Flight Scenario}

The validation of the autopilot involves several steps, from robustness analysis and simulations on LTI models corresponding to different equilibrium points to simulations of complete flight scenarios on the full nonlinear model. For brevity, only this last step is presented.

A representative flight scenario is considered, with launch conditions defined by the initial velocity, $V_{0}=939 \mathrm{~m} / \mathrm{s}$, elevation $\theta_{0}=42^{\circ}$, and azimuth $\psi_{0}=0^{\circ}$. The flight of the guided projectile is made of several phases. Initially, the GNC components are set to be inactive because of the particularly harsh launch conditions. At $t=20 \mathrm{~s}$, roll rate reduction of the forward part is initiated by sending a constant command signal $\delta_{p}=10^{\circ}$ to the actuators, and once the roll angular rate $p_{f}$ is down to 5 turns per second, the roll-channel autopilot designed in [6] stabilises the roll angle at $45^{\circ}$. The roll rate reduction and roll control phases last about $0.5 \mathrm{~s}$. At $t=30 \mathrm{~s}$, the pitch/yaw GNC components are activated, and the projectile enters its guided phase until impact.

The target is set to be the ballistic impact point of the projectile. The position of the target is taken into account in the closed-loop through a zero-effort-miss (ZEM) guidance law described in [11], which provides reference normal and lateral load factors $n_{z, c}$ and $n_{y, c}$ sent to the controller. Simulation results are shown in Fig. 7. It can be observed that the load factors are well followed, and the target is successfully reached with very high accuracy. Furthermore, the deflection angles remain below the saturation level of $\pm 10^{\circ}$ for the whole duration of the guided phase.

\section{Motivating Scenario for Anti-Windup}

Referring to the scenario presented in Section III-C, suppose now that the initial conditions differ from their nominal values as follows:

$$
V_{0}=931.6 \mathrm{~m} / \mathrm{s} \quad \theta_{0}=42.1^{\circ} \quad \psi_{0}=-0.004^{\circ}
$$



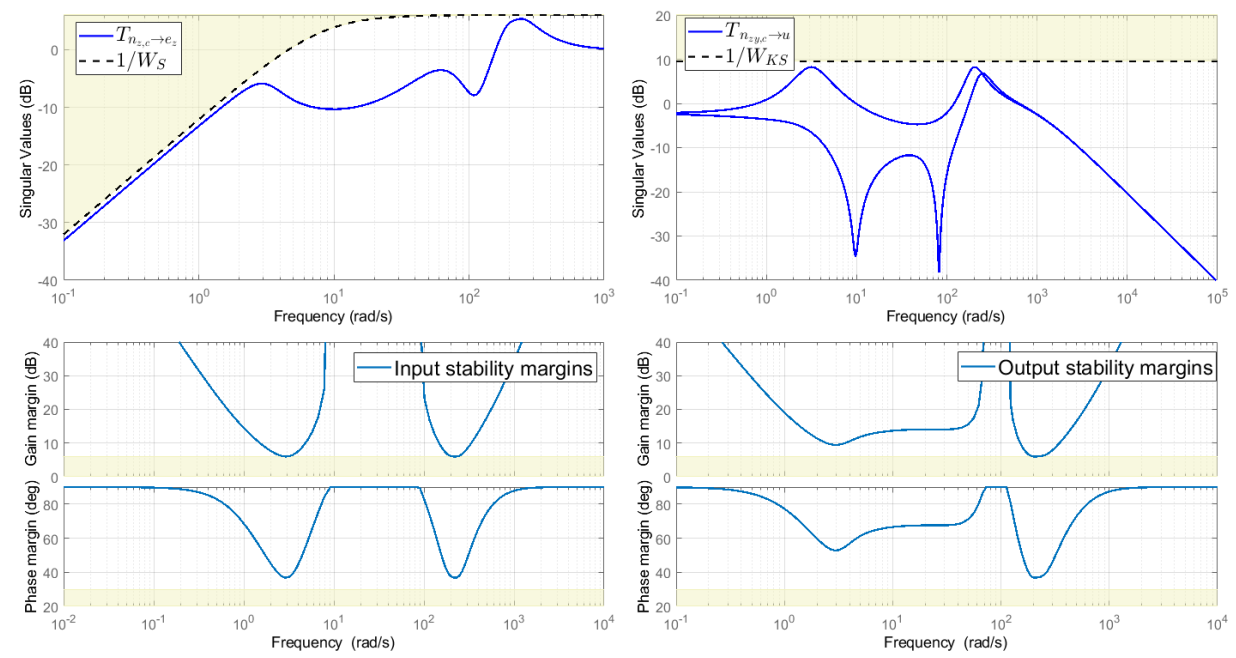

Fig. 3. Shaped closed-loop transfer functions and stability margins at controller input and output
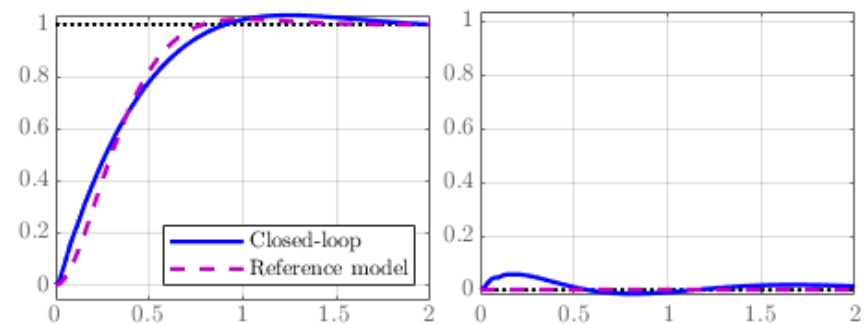

Fig. 4. Closed-loop response in $n_{z}$ (left) and $n_{y}$ (right) to a step input on $n_{z, c}$

while the target position remains unchanged. The resulting guidance and command signals along with the associated trajectory are presented in Fig. 8 for inactive and active saturations. It can be seen that, ignoring saturations, the baseline autopilot manages to correct the misconfiguration of the cannon, at the price of commanding slightly higher deflection angles. However, as seen from the results with active saturations, this is enough to completely disrupt the behaviour of the guidance, and hence the whole guided flight, once saturations occur. Ultimately, the projectile miss distance goes from a satisfying value of $4 \cdot 10^{-3} \mathrm{~m}$ to $85.4 \mathrm{~m}$.

\section{Anti-Windup Augmentation}

Following the results of the previous section, the antiwindup problem is now addressed. The general principle of anti-windup compensation in the LTI context is illustrated by Fig. 5 (see e.g. [12]). The output $v=\left[v_{1}, v_{2}\right]^{T}$ of the antiwindup device modifies the controller dynamics as follows:

$$
\left\{\begin{array}{l}
\dot{x}_{c}=A_{c} x_{c}+B_{c}\left[\begin{array}{c}
y \\
w
\end{array}\right]+v_{1} \\
y_{c}=C_{c} x_{c}+D_{c}\left[\begin{array}{c}
y \\
w
\end{array}\right]+v_{2}
\end{array}\right.
$$

where $\left(A_{c}, B_{c}, C_{c}, D_{c}\right)$ is a state-space representation of the controller. The exogenous input $w$ represents here the reference signal $w=\left[n_{z, c}, n_{y, c}\right]^{T}$. It should be noted that the output of the controller as designed in Section III contains the virtual signals $\left[\delta_{q}, \delta_{r}\right]$. However, as mentioned in Section II, the saturations apply to the real canard deflections $\left[\delta_{i}\right]_{i=1 \ldots 4}$.

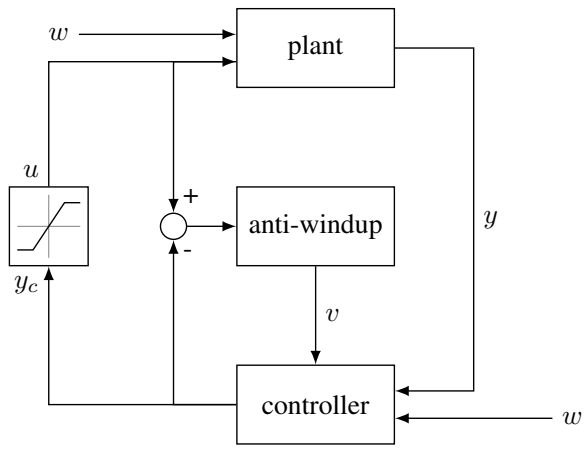

Fig. 5. Principle of anti-windup compensation

Assuming a fixed nose roll angle $\phi_{f}=45^{\circ}$ (ensured by the roll autopilot), Equation (3) leads to a saturation level $\delta_{\text {sat,virtual }}=\sqrt{2} \times 10^{\circ}$ applying on the signals $\delta_{q}+\delta_{r}$ and $\delta_{q}-\delta_{r}$. This motivates defining the controller output as $y_{c}=\left[\delta_{q}+\delta_{r}, \delta_{q}-\delta_{r}\right]^{T}$ in the anti-windup problem. Further simulations using standard launch conditions show that this orientation of the nose is advantageous to reduce the occurrence of saturations, and in particular outperforms the other standard configuration characterised by $\phi_{f}=0^{\circ}$.

To minimise the on-board computational burden, a static anti-windup is considered for the projectile, with furthermore $v_{2}=0$ to avoid algebraic loops. Thus anti-windup synthesis amounts to computing the matrix gain $D_{a w} \in \mathbb{R}^{2 \times 2}$ such that:

$$
v_{1}=D_{a w}\left[\begin{array}{l}
\operatorname{sat}\left(\delta_{q}+\delta_{r}\right)-\left(\delta_{q}+\delta_{r}\right) \\
\operatorname{sat}\left(\delta_{q}-\delta_{r}\right)-\left(\delta_{q}-\delta_{r}\right)
\end{array}\right]
$$

This computation is done using the SAW library of the SMAC toolbox (http://w3.onera.fr/smac) so as to maximise the amplitude of step input reference signals $w$ for which stability can be guaranteed. The implemented algorithms are described in [13] and [14]. They rely on an LMIbased Lyapunov approach, exploiting the modified sector condition of [15] to characterise the deadzone nonlinearity, and using autonomous systems of the form:

$$
\dot{w}=-\lambda w \quad w(0)=w_{0}
$$

to model $\mathcal{L}_{2}$ bounded step-like reference inputs of amplitude 


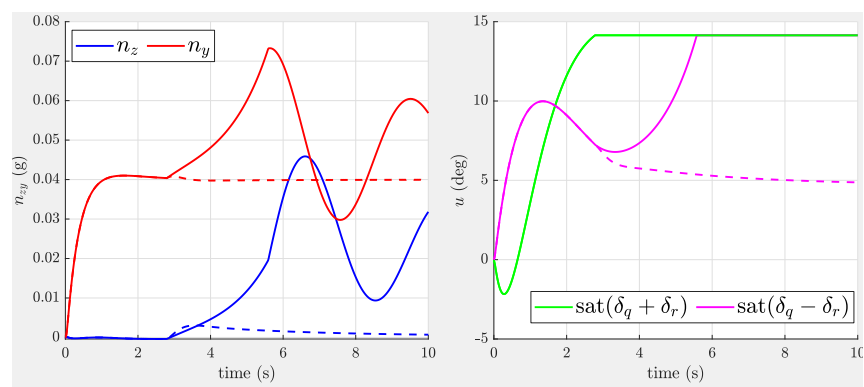

Fig. 6. Response to a step input on $n_{y}$ : without anti-windup (full line) and with anti-windup (dashed)

$\left\|w_{0}\right\|$. Fig. 6 shows how the computed anti-windup compensator can greatly improve the step response of the saturated closed-loop at a design point, for amplitudes of the order of magnitude of what is expected during the trajectory.

The parameter-dependent nature of the problem is tackled in the same way as for the baseline, i.e. an anti-windup compensator is computed for each point of a grid of the reduced flight envelope, with a linear interpolation yielding the global compensator. Fig. 9 shows the guidance and control signals along with the projectile trajectory for the scenario described in Section III-D when the autopilot is augmented with the computed anti-windup, and clearly illustrates the potential of the approach in recovering high accuracy when the baseline autopilot alone fails.

\section{Simulation Results}

In this section, the nonlinear closed-loop with and without anti-windup compensation are compared through Monte Carlo trajectory simulations. These are performed by considering uncertainties on the launch conditions. The samples are drawn following normal distributions whose characteristics are detailed in Table I, and which include the problematic case that was presented in Section III-D.

Out of 500 simulations, 19 (3.8\%) involve command signals reaching the saturation level, making it a relatively rare but non-negligible event. For all the others, the antiwindup compensator remains inactive all along the trajectory, and the combined action of the ZEM guidance and baseline autopilot ensures very good accuracy at impact. Over these 19 simulations, the miss distance using the baseline autopilot averages at $30.2 \mathrm{~m}$ with a maximum value of $130.6 \mathrm{~m}$. For reference, the miss distance in a ballistic configuration lies between 250 and $350 \mathrm{~m}$. The anti-windup compensator corrects all but two cases, which nevertheless display a greatly reduced miss distance $(8.3 \mathrm{~m}$ and $17.7 \mathrm{~m})$. With respect to the full sample used for the Monte Carlo simulations, this brings the success rate of the guided projectile with anti-windup augmentation to $99.6 \%$ against uncertain initial conditions.

\begin{tabular}{cccc}
\hline Parameter & Mean value & Standard deviation & Unit \\
\hline$V_{0}$ & 939 & 3 & $\mathrm{~m} / \mathrm{s}$ \\
$\theta_{0}$ & 42 & 0.09 & $\circ$ \\
$\psi_{0}$ & 0 & 0.12 & $\circ$ \\
\hline
\end{tabular}

TABLE I

UnCERTAinties USED FOR MONTE CARLO TRAJECTORIES

\section{CONCLUSION}

This article addresses the problem of controlling a canardguided dual-spin projectile. The design of a gain-scheduled baseline autopilot is presented, which successfully ensures high terminal accuracy in nominal flight conditions. However, the need for anti-windup compensation arises when considering perturbed launch conditions. A simple gainscheduled anti-windup compensator is proposed, and simulation results show that the augmented closed-loop is capable of recovering high performance in most cases.

Future work will focus on taking into account additional uncertainties and disturbances (aerodynamic uncertainties, wind, sensor delays and noise). These are more challenging as their effect on the closed-loop dynamics can be significant. More sophisticated anti-windup schemes may be considered to further increase the operational domain of the system. IQC-based robustness analysis methods will also be integrated in the design process to compare anti-windup schemes and alleviate the need for intensive simulations.

\section{REFERENCES}

[1] S. Theodoulis, F. Sève, and P. Wernert, "Robust gain-scheduled autopilot design for spin-stabilized projectiles with a course-correction fuze," Aerospace Science and Technology, 2015.

[2] F. Sève, S. Theodoulis, P. Wernert, M. Zasadzinski, and M. Boutayeb, "Flight dynamics modeling of dual-spin guided projectiles," IEEE Transactions on Aerospace and Electronic Systems, 2017.

[3] F. Sève, S. Theodoulis, P. Wernert, M. Zasadzinski, and M. Boutayeb, "Gain-scheduled $\mathcal{H}_{\infty}$ loop-shaping autopilot design for spin-stabilized canard-guided projectiles," AerospaceLab Journal, 2017.

[4] S. Theodoulis, V. Gassmann, T. Brunner, and P. Wernert, "Fixed structure robust control design for the $155 \mathrm{~mm}$ canard-guided projectile roll-channel autopilot," in Proceedings of the 21st Mediterranean Conference on Control and Automation, 2013.

[5] P. H. Zipfel, Modeling and Simulation of Aerospace Vehicle Dynamics, 2nd ed. AIAA Education Series, 2007.

[6] S. Thai, S. Theodoulis, C. Roos, and J.-M. Biannic, "Robust design for the roll-channel autopilot of a canard-guided dual-spin projectile," IFAC-PapersOnLine, 2019, 21st IFAC Symposium on Automatic Control in Aerospace ACA 2019.

[7] W. J. Rugh and J. S. Shamma, "Research on gain scheduling," Automatica, 2000.

[8] P. Apkarian and D. Noll, "Nonsmooth $H_{\infty}$ synthesis," IEEE Transactions on Automatic Control, 2006.

[9] S. Skogestad and I. Postlethwaite, Multivariable Feedback Control: Analysis and Design, 2nd ed. Wiley, 2005.

[10] P. Apkarian, D. Noll, and A. M. Simoes, "Time-domain control design: A nonsmooth approach," IEEE Transactions on Control Systems Technology, 2009.

[11] M. Proff and S. Theodoulis, "Study of impact point prediction methods for zero-effort-miss guidance: Application to a $155 \mathrm{~mm}$ spin-stabilized guided projectile," in Proceedings of the 5th CEAS Conference on Guidance, Navigation and Control, 2019.

[12] S. Galeani, S. Tarbouriech, M. Turner, and L. Zaccarian, "A tutoria on modern anti-windup design," European Journal of Control, 2009.

[13] J.-M. Biannic and S. Tarbouriech, "Optimization and implementation of dynamic anti-windup compensators with multiple saturations in flight control systems," Control Engineering Practice, 2009.

[14] C. Roos and J.-M. Biannic, "A convex characterization of dynamicallyconstrained anti-windup controllers," Automatica, 2008.

[15] J. M. G. da Silva and S. Tarbouriech, "Antiwindup design with guaranteed regions of stability: an LMI-based approach," IEEE Transactions on Automatic Control, 2005. 

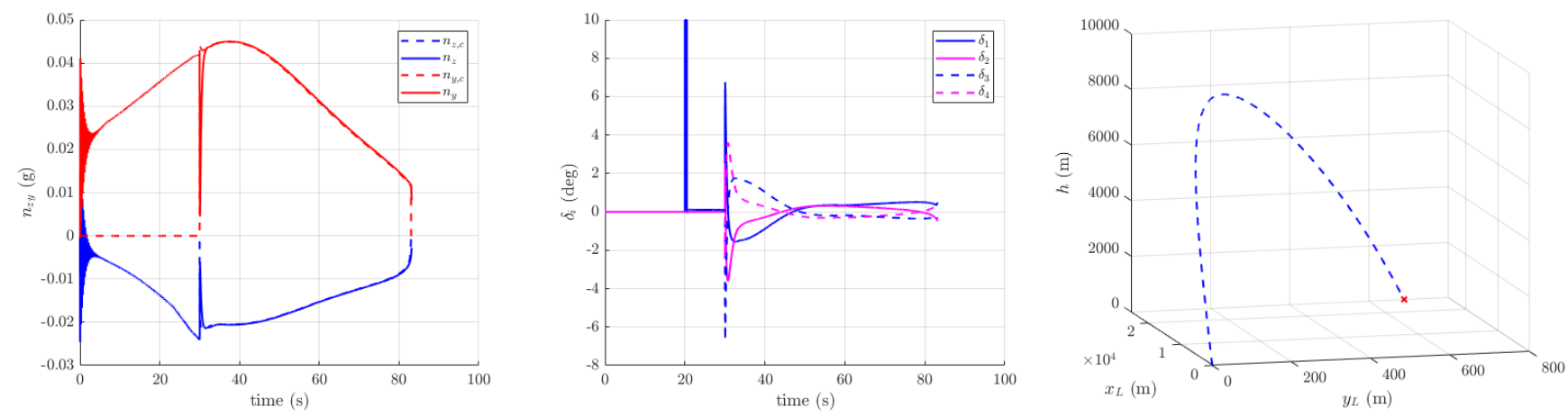

Fig. 7. Nominal flight scenario: normal and lateral accelerations (left), canard deflection angles (middle), trajectory (right)
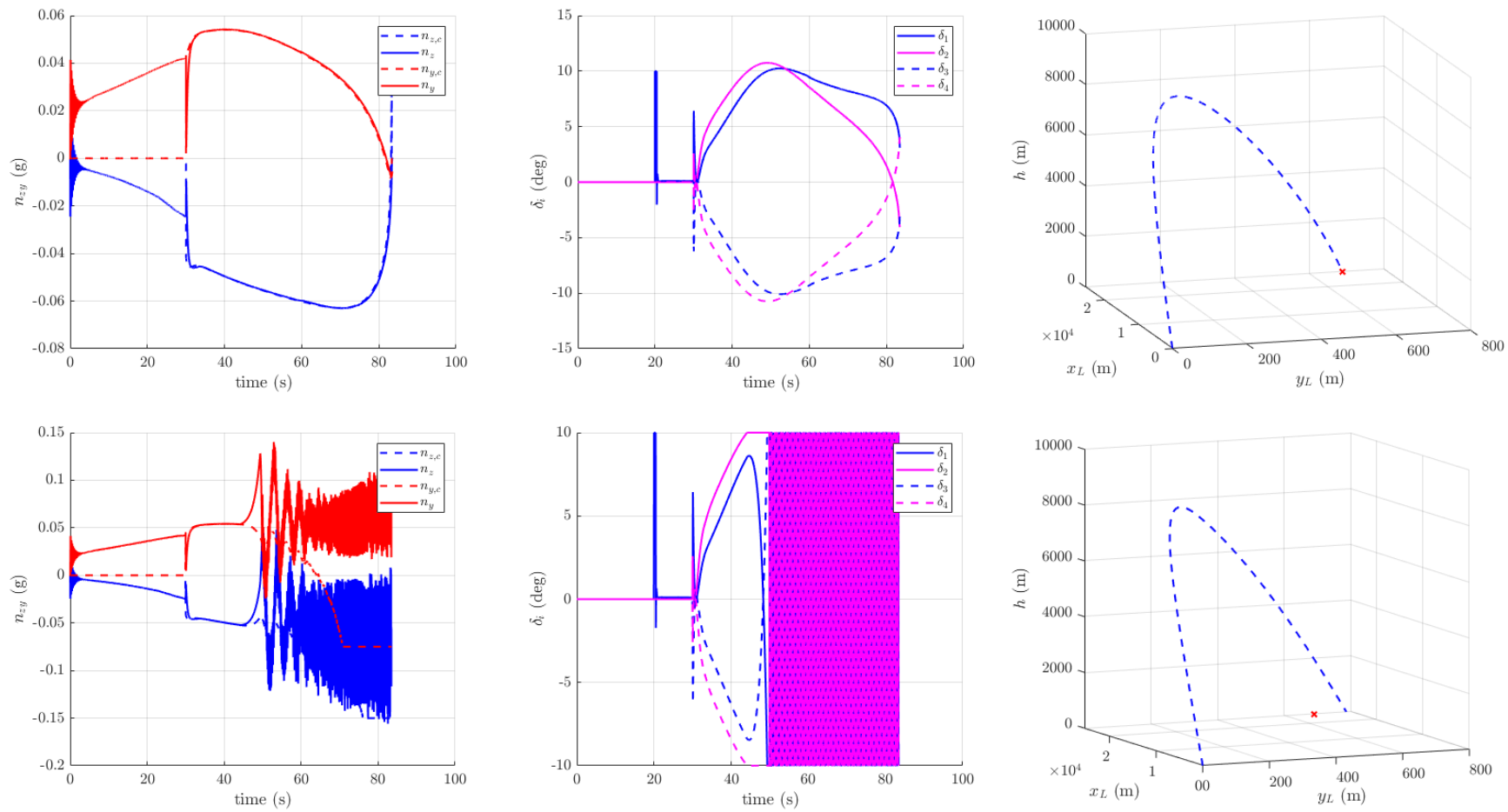

Fig. 8. Degraded initial conditions without saturations (top) and with saturations (bottom); normal and lateral accelerations (left), canard deflection angles (middle), trajectory (right)
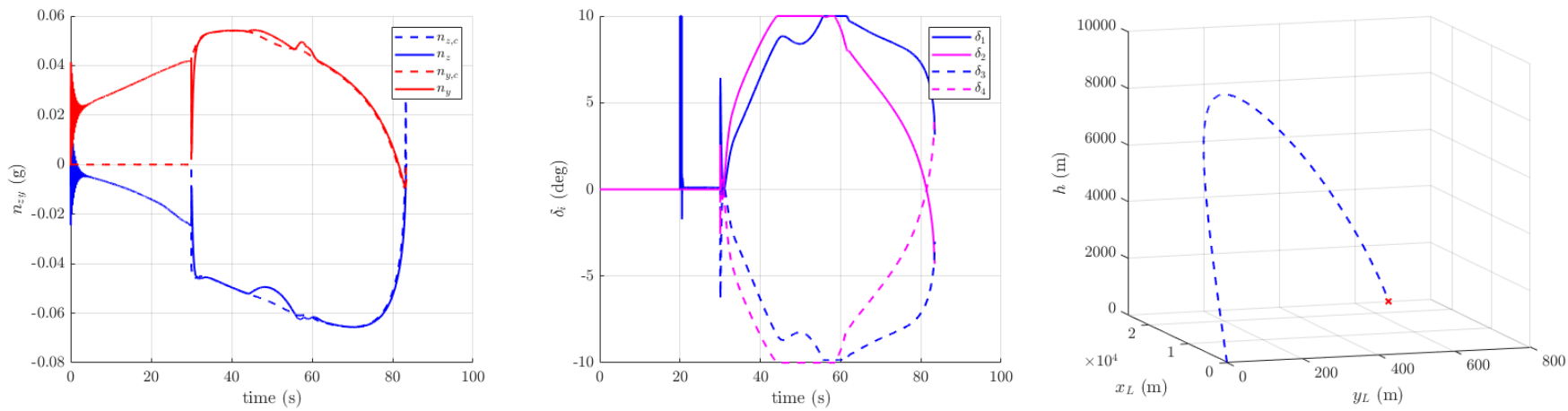

Fig. 9. Degraded initial conditions with saturations and anti-windup compensation: normal and lateral accelerations (left), canard deflection angles (middle), trajectory (right) 